\title{
Contour Plots of Analytic Functions
}

\section{Report}

Author(s):

Gautschi, Walter; Waldvogel, Jörg

Publication date:

1996-10

Permanent link:

https://doi.org/10.3929/ethz-a-004284538

Rights / license:

In Copyright - Non-Commercial Use Permitted

Originally published in:

SAM Research Report 1996-15 


\title{
Contour Plots of Analytic Functions
}

\author{
W. Gautschi ${ }^{1}$ and J. Waldvogel
}

Research Report No. 96-15

October 1996

Seminar für Angewandte Mathematik

Eidgenössische Technische Hochschule

CH-8092 Zürich

Switzerland

${ }^{1}$ Computer Science Department, Purdue University, West Lafayette, IN 47907, USA 


\title{
Contour Plots of Analytic Functions
}

\author{
W. Gautschi ${ }^{1}$ and J. Waldvogel \\ Seminar für Angewandte Mathematik \\ Eidgenössische Technische Hochschule \\ CH-8092 Zürich \\ Switzerland
}

Research Report No. 96-15 October 1996

\begin{abstract}
This is a tutorial on generating contour lines of an analytic function $f(z)$. The emphasis is on using mathematical software (MATLAB, to a lesser extent MAPLE) for implementing the algorithms, and efficient programs together with explanations are presented. Two different approaches are suggested: (1) generating level lines as contours of, e.g., constant modulus or constant phase of the function $f(z),(2)$ setting up and numerically integrating an appropriate differential equation for the contour under consideration. Both methods are demonstrated by means of the $n$th partial sum $f(z)=e_{n}(z)$ of the exponential series. The line of constant modulus satisfying $\left|e_{n}(z)\right|=1$ has a practical significance: it delineates the region of absolute stability for an explicit Taylor integrator of order $n$.
\end{abstract}

\footnotetext{
${ }^{1}$ Computer Science Department, Purdue University, West Lafayette, IN 47907, USA. Supported, in part, by the National Science Foundation under grant DMS-9305430
} 


\section{Introduction}

There are two easy ways in MATLAB to construct contour plots of analytic functions, i.e., lines of constant modulus and constant phase. One is to use the MATLAB contour command for functions of two variables, another to solve the differential equations satisfied by the contour lines. This is illustrated here for the function $f(z)=e_{n}(z)$, where

$$
e_{n}(z)=1+z+\frac{z^{2}}{2 !}+\cdots+\frac{z^{n}}{n !}
$$

is the $n$th partial sum of the exponential series. The lines of constant modulus 1 of $e_{n}$ are of interest in the numerical solution of ordinary differential equations, where for $1 \leq n \leq 4$ they delineate regions of absolute stability of explicit one-step methods of order $n$ (cf. [4, §9.3.2]). For $n \geq 1$ the 1-line of $e_{n}$ delineates the region of absolute stability of the explicit Taylor method of order $n$.

\section{Contour Plots by the contour Command}

Let $f$ be analytic and $f(z)=r e^{i \varphi}$. We may consider the modulus $r$ as a function of two variables $x, y$, where $z=x+i y$; similarly for the phase $\varphi,-\pi<\varphi \leq \pi$. Hence, we can apply the MATLAB command contour to $r$ and $\varphi$ to obtain the lines of constant modulus and phase.

In the MATLAB program below, the set of all $x$ - and $y$-values is collected (in true MATLAB spirit) in a matrix a, which is operated upon to compute the desired values of $r$ and $\varphi$ for $f=e_{n}$ as input matrices to the routine contour.

The program begins with the definitions of the mesh $\mathrm{h}$ and the number nmax of contour plots to be generated. The vector bounds contains common lower and upper bounds for the $x$ - and $y$-coordinates applicable for all plots. The bounds used here have been chosen to accommodate contour plots of the first four exponential sums. Then the contour levels vabso and vango for the modulus and phase of $f(z)$ are defined. The last preparatory step is generating the vectors $\mathrm{x}$ and $\mathrm{y}$ containing the discrete $x$ - and $y$-values to be used in the matrix a of grid points. In the loop over $n$ the values $f$ of $e_{n}$ on the entire grid are generated by almost the same statements that would evaluate $e_{n}$ at a single point, where t stands for an individual term of the series (1). The only difference is the statement $t=t . * a / n$, in which the operation symbol .* invokes the element-by-element product of the matrices $t$ and $a$. The last line of the program (here turned off by the comment sign \%) generates the encapsulated postscript file fign.eps of figure(n), ready to be printed or incorporated into a text file.

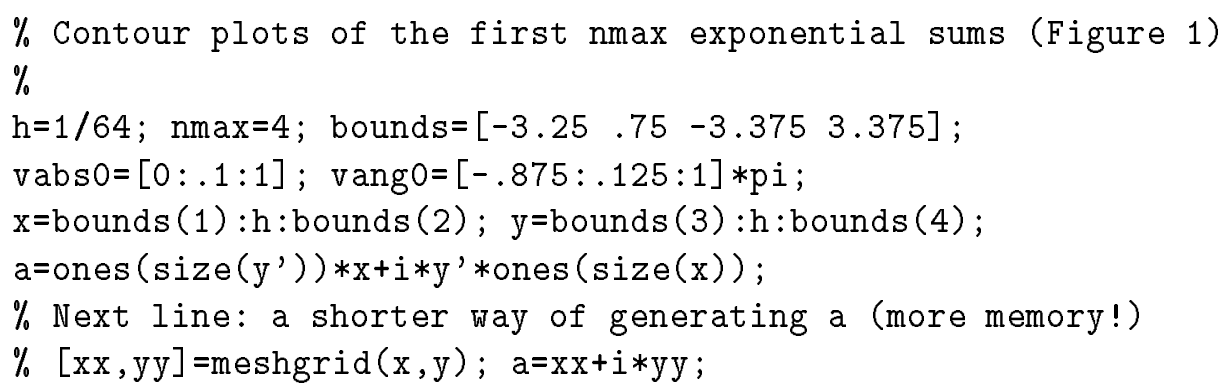




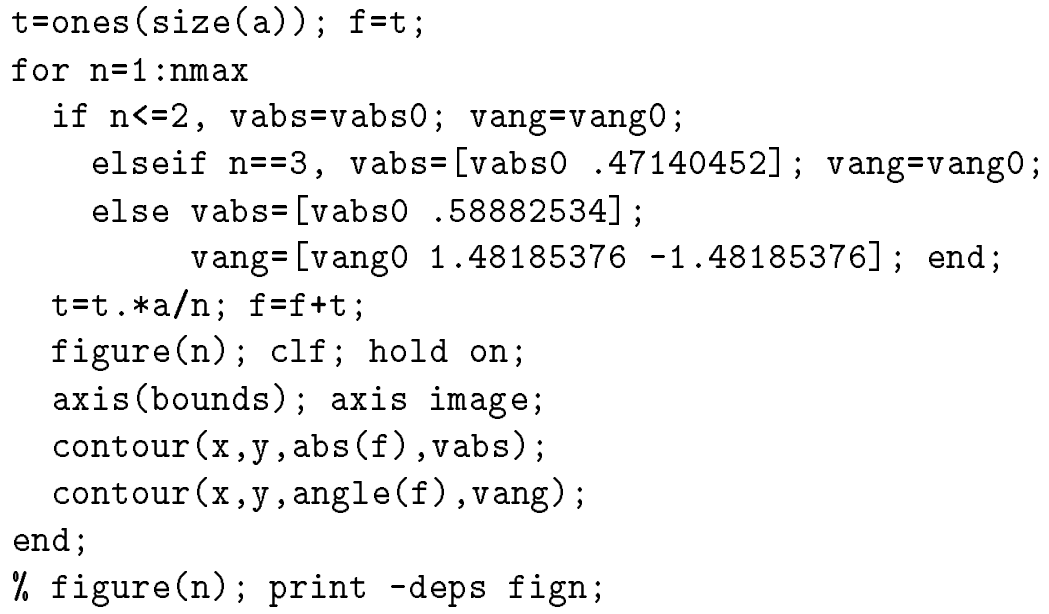

The results for $n=1: 1: 4^{1}$ are shown in the plots below. Clearly visible are the $n$ zeros of $e_{n}$ from which emanate the lines of constant phase. Near these zeros, the lines of constant modulus become circle-like with radii tending to 0 as the zeros are approached. The contour lines are for $r=.1: .1: 1$ and $\varphi=-\frac{7}{8} \pi: \frac{1}{8} \pi: \pi$.

At points $z_{0}$ where $e_{n}^{\prime}\left(z_{0}\right)=e_{n-1}\left(z_{0}\right)=0, n \geq 2$, two lines of constant modulus intersect (cf. $\S 3.1$ below). The respective $r$-values are $r=\left|e_{n}\left(z_{0}\right)\right|$, or $r=\left|z_{0}\right|^{n} / n !$, since

$$
e_{n}(z)=e_{n-1}(z)+\frac{z^{n}}{n !}
$$

These critical lines are also included in the plots (see the if statement of the program). When $n=2$, they go through $z_{0}=-1$, where $r=\frac{1}{2}$, while for $n=3$ and $n=4$, one has to 8 decimal digits: $z_{0}=-1 \pm i, r=\sqrt{2} / 3=.47140452$ and $z_{0}=-.70196418 \pm 1.80733949 i$, $r=(1.93887332)^{4} / 24=.58882535$, respectively.

What's good for the $r$-lines is good for the $\varphi$-lines! The singular points for them are also the zeros $z_{0}$ of $e_{n}^{\prime}$ (cf. $\S 3.2$ ), to which there correspond $\varphi$-values defined by $e_{n}\left(z_{0}\right) /\left|e_{n}\left(z_{0}\right)\right|=$ $\left(z_{0} /\left|z_{0}\right|\right)^{n}=e^{i \varphi}$, i.e., $\varphi=n \arg z_{0}$. Thus, for $n=2$, we have $\varphi=0(\bmod 2 \pi)$, whereas for $n=3$ we get $\varphi= \pm \frac{\pi}{4}$ corresponding to $z_{0}=-1 \pm i$, respectively. All three of these $\varphi$-values are included among the values already listed above. For $n=4$, the two values of $z_{0}$ shown in the previous paragraph yield $\varphi=4 \arg z_{0}= \pm 1.48185376(\bmod 2 \pi)$. These critical $\varphi$-lines are also shown in the plots in Figure 1.

The figure was generated by means of the step size $\mathrm{h}=1 / 64$ in order to obtain a good resolution, even for the "branch cuts" corresponding to $\mid$ angle(f) $\mid=p i$. The choice $h=1 / 32$ is a good compromise, whereas $\mathrm{h}=1 / 16$ is very fast while still producing satisfactory plots.

\footnotetext{
${ }^{1}$ This MATLAB notation stands for $n=1,2,3,4$.
} 
Figure 1. Contour Plots of the First 4 Exponential Sums
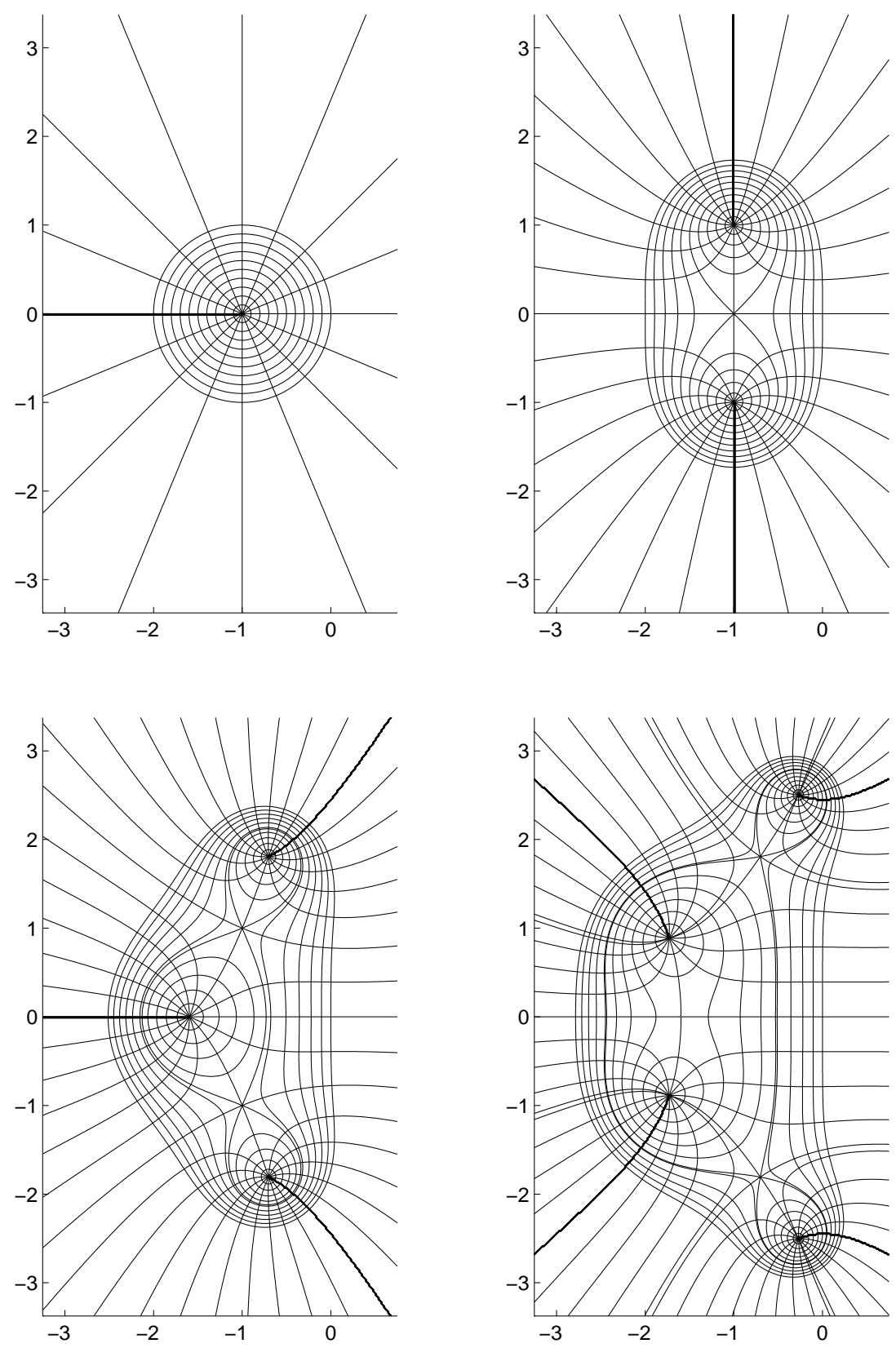

\section{Differential Equations}

For an analytic function $f$, let

$$
w=f(z), \quad w=r e^{i \varphi}, \quad z=x+i y .
$$

3.1. Contour lines $r=$ const. To describe the lines $r=$ const, it is natural to take $\varphi$ as independent variable. Differentiating

$$
f(z(\varphi))=r e^{i \varphi}, \quad r=\text { const },
$$


with respect to $\varphi$ then gives $f^{\prime}(z) \frac{d z}{d \varphi}=i r e^{i \varphi}=i f(z)$, that is,

$$
\frac{d z}{d \varphi}=i q(z), \quad \text { where } \quad q(z)=\frac{f(z)}{f^{\prime}(z)}
$$

With $s$ the arc length, one has

$$
\frac{d s}{d \varphi}=\sqrt{\left(\frac{d x}{d \varphi}\right)^{2}+\left(\frac{d y}{d \varphi}\right)^{2}}=\left|\frac{d z}{d \varphi}\right|=|q(z)|,
$$

so that

$$
\frac{d z}{d s}=\frac{d z}{d \varphi} \frac{d \varphi}{d s}=i \frac{q(z)}{|q(z)|}
$$

Written as a system of differential equations, this is

$$
\begin{aligned}
& \frac{d x}{d s}=-\operatorname{Im}\left\{\frac{q(z)}{|q(z)|}\right\}, \\
& \frac{d y}{d s}=\operatorname{Re}\left\{\frac{q(z)}{|q(z)|}\right\},
\end{aligned}
$$

If we are interested in a contour line crossing the real axis, we must find an initial point $x(0)=x_{r}, y(0)=0$ for $(7)$ with real $x_{r}$ such that $f\left(x_{r}\right)=r$ (assuming $f(x)$ real for real $\left.x\right)$. In the case $f(x)=e_{n}(x)$, this is easy if $r \geq 1$, since $e_{n}(0)=1$ and $e_{n}(x)$ monotonically increases for $x \geq 0$. There is thus a unique $x_{r} \geq 0$ such that $e_{n}\left(x_{r}\right)=r$. If $0<r<1$, this is still possible when $n$ is odd. Then, $e_{n}^{\prime}(x)=e_{n-1}(x)>0$, since all zeros of $e_{m}$, when $m$ is even, are known to be complex [3] (cf. also [1]). Thus, $e_{n}$ monotonically increases from $-\infty$ to $+\infty$ as $x$ increases from $-\infty$ to $+\infty$, and there is a unique $x_{r}<0$ such that $e_{n}\left(x_{r}\right)=r$. When $n$ is even, we have $e_{n}(x)>0$ for all real $x$, and $\epsilon_{n}^{\prime}=e_{n-1}$ vanishes at exactly one point $x_{0}<0$, where $e_{n}$ has a minimum (cf. [2]). Owing to (2) and $e_{n-1}\left(x_{0}\right)=0$, we have $e_{n}\left(x_{0}\right)=x_{0}^{n} / n !$, and there is a solution $x_{r}<0$ of $e_{n}\left(x_{r}\right)=r$ if and only if $r \geq x_{0}^{n} / n$ !. For smaller positive values of $r$, one must find a complex initial point $x(0), y(0)>0$ near one of the complex zeros of $e_{n}$.

The point $z_{0}$ where $f^{\prime}\left(z_{0}\right)=0$ is a singular point of $(7)$, a point where two $r$-lines intersect at a right angle. This requires special care to get the integration of ( 7$)$ started in all four directions. The initial point, of course, is $z_{0}$, that is, $x(0)=\operatorname{Re} z_{0}, y(0)=\operatorname{Im} z_{0}$. What needs some analysis is the value of the right-hand side of $(7)$ at $z_{0}$. Let $h(z)=\left(z-z_{0}\right) q(z)$; then $h$ is smooth near $z_{0}$ and has the Taylor expansion

$$
h(z)=\frac{f_{0}+\frac{1}{2}\left(z-z_{0}\right)^{2} f_{0}^{\prime \prime}+\cdots}{f_{0}^{\prime \prime}+\frac{1}{2}\left(z-z_{0}\right) f_{0}^{\prime \prime \prime}+\cdots}, \quad h\left(z_{0}\right)=\frac{f_{0}}{f_{0}^{\prime \prime}},
$$

where $f_{0}=f\left(z_{0}\right)$, etc. (we assume $f_{0} \neq 0$ and $f_{0}^{\prime \prime} \neq 0$ ). Letting

$$
\frac{z-z_{0}}{\left|z-z_{0}\right|}=e^{i \theta}, \quad-\frac{1}{2} \pi<\theta \leq \frac{1}{2} \pi, \quad h\left(z_{0}\right) /\left|h\left(z_{0}\right)\right|=e^{i \omega}, \quad-\pi<\omega \leq \pi,
$$

(being mindful that to each $\theta$ there is a $\theta+\pi$ corresponding to the backward continuation of the line), we then have

$$
\frac{q(z)}{|q(z)|}=\frac{\left|z-z_{0}\right|}{z-z_{0}} \frac{h(z)}{|h(z)|} \rightarrow e^{i\left(\omega-\theta_{0}\right)} \quad \text { as } z \rightarrow z_{0},
$$


where $\theta_{0}=\lim _{z \rightarrow z_{0}} \theta$. It remains to determine $\theta_{0}$.

Along an $r$-line through $z_{0}$, we have

$$
\begin{aligned}
r^{2} & =|f(z)|^{2}=\left|f_{0}+\frac{1}{2}\left(z-z_{0}\right)^{2} f_{0}^{\prime \prime}+\cdots\right|^{2} \\
& =\left|f_{0}\right|^{2}+\operatorname{Re}\left[\left(z-z_{0}\right)^{2} f_{0}^{\prime \prime} \overline{f_{0}}\right]+O\left(\left|z-z_{0}\right|^{3}\right) .
\end{aligned}
$$

Since $\left|f_{0}\right|^{2}=r^{2}$, this gives

$$
\operatorname{Re}\left\{\left(\frac{z-z_{0}}{\left|z-z_{0}\right|}\right)^{2} f_{0}^{\prime \prime} \overline{f_{0}}\right\}=O\left(\left|z-z_{0}\right|\right)
$$

hence, as $z \rightarrow z_{0}$,

$$
\operatorname{Re}\left(e^{2 i \theta_{0}} f_{0}^{\prime \prime} \overline{f_{0}}\right)=0
$$

Therefore,

$$
\tan 2 \theta_{0}=\frac{\operatorname{Re}\left(f_{0}^{\prime \prime} \overline{f_{0}}\right)}{\operatorname{Im}\left(f_{0}^{\prime \prime} \overline{f_{0}}\right)}
$$

There are exactly two solutions in $-\frac{1}{2} \pi<\theta_{0} \leq \frac{1}{2} \pi$, which differ by $\frac{1}{2} \pi$, confirming the orthogonality of the two $r$-lines through $z_{0}$.

Note that in the case $f(z)=e_{n}(z)$, we have $f^{\prime}(z)=e_{n-1}(z)$, so that $z_{0}$ is a zero of $e_{n-1}$. This is clearly visible in the plots of $\S 2$. Furthermore, $f_{0}=e_{n}\left(z_{0}\right), f_{0}^{\prime \prime}=e_{n-2}\left(z_{0}\right)$ if $n \geq 2$, so that (2) with $z=z_{0}$, once applied as is, and once with $n$ replaced by $n-1$, gives $f_{0}=z_{0}^{n} / n$ !, $f_{0}^{\prime \prime}=-z_{0}^{n-1} /(n-1) !$, and the equation for $\theta_{0}$ reduces to

$$
\tan 2 \theta_{0}=-\frac{\operatorname{Re} z_{0}}{\operatorname{Im} z_{0}} \quad\left(f=e_{n}\right) .
$$

3.2. Contour lines $\varphi=$ const. For the lines $\varphi=$ const, we take $r$ as the independent variable and, by differentiating

$$
f(z(r))=r e^{i \varphi}, \quad \varphi=\text { const }
$$

with respect to $r$, obtain

$$
\frac{d z}{d r}=\frac{e^{i \varphi}}{f^{\prime}(z)} .
$$

In terms of the arc length $s$, we now have

$$
\frac{d s}{d r}=\left|\frac{d z}{d r}\right|=\frac{1}{\left|f^{\prime}(z)\right|},
$$

so that

$$
\frac{d z}{d s}=\frac{d z}{d r} \frac{d r}{d s}=e^{i \varphi} \frac{\left|f^{\prime}(z)\right|}{f^{\prime}(z)},
$$

or, written as a system of differential equations,

$$
\begin{aligned}
& \frac{d x}{d s}=\operatorname{Re}\left\{e^{i \varphi} \frac{\left|f^{\prime}(z)\right|}{f^{\prime}(z)}\right\}, \quad z=x+i y . \\
& \frac{d y}{d s}=\operatorname{Im}\left\{e^{i \varphi} \frac{\left|f^{\prime}(z)\right|}{f^{\prime}(z)}\right\},
\end{aligned}
$$


The singular point of $(9)$ is again $z_{0}$, a zero of $f^{\prime}$. At this point,

$$
\frac{f_{0}}{\left|f_{0}\right|}=e^{i \varphi}, \quad-\pi<\varphi \leq \pi
$$

which determines $\varphi$. The limit of $\left|f^{\prime}(z)\right| / f^{\prime}(z)$ as $z \rightarrow z_{0}$ may be determined by a procedure similar to the one in $\$ 3.1$. Instead, we directly use the Taylor series of $f$ in $z_{0}$ in order to study the $\varphi$-lines (and the $r$-lines as well) near $z_{0}$ with $f^{\prime}\left(z_{0}\right)=0$. Let $z=z_{0}+\zeta$, where $\zeta$ is a complex increment, and let

$$
f_{k}:=f^{(k)}\left(z_{0}\right), \quad k \geq 0, \quad f_{0} \neq 0, \quad f_{1}=0, \quad f_{2} \neq 0,
$$

be the derivatives of $f$ at $z_{0}$. Then the Taylor series is

$$
f\left(z_{0}+\zeta\right)=f_{0}+f_{2} \frac{\zeta^{2}}{2 !}+f_{3} \frac{\zeta^{3}}{3 !}+\cdots
$$

Next, we observe that by defining $w=f_{0} e^{u}$ in (3), i.e., by putting

$$
f\left(z_{0}+\zeta\right)=f\left(z_{0}\right) e^{u}
$$

the $r$-lines through $z_{0}$ are given by the values of $\zeta$ corresponding to purely imaginary values $u=i t$, whereas the $\varphi$-lines through $z_{0}$ are given by $u \in R$. The point $z_{0}$ itself corresponds to $\zeta=u=0$. We therefore need to solve Equ. (12), with $f\left(z_{0}+\zeta\right)$ substituted from (11), for $\zeta$, which is a typical task for MAPLE.

In the program below ${ }^{2}$ the series (11) and the equation (12) are denoted by $\mathbf{s}$ and eq, respectively. The solve command automatically expands $e^{u}$ in a Taylor series and solves the equation by means of a series progressing in appropriate powers of $u$ (here half-integer powers). As expected, two solutions corresponding to the two possible values of the square root are found. Only the first solution tt zet0[1] is processed further: first, by substituting the abbreviations $f \mathrm{k}$ defined in Equ. (10), then by introducing the variable v according to

$$
u=\frac{v^{2} f_{2}}{2 f_{0}} \quad \text { or } \quad v=\left(\frac{2 u f_{0}}{f_{2}}\right)^{\frac{1}{2}} .
$$

The symbols $\mathrm{D}(\mathrm{f})$ and (D@@k) (f) stand for the derivative of $f$ and the $k$ th derivative of $f$, respectively. The call to the function map causes the operation defined by its first argument, here the simplification of the radicals, to be applied to each term of the expression defined by the second argument. Finally, the call to series causes the O-term to be simplified.

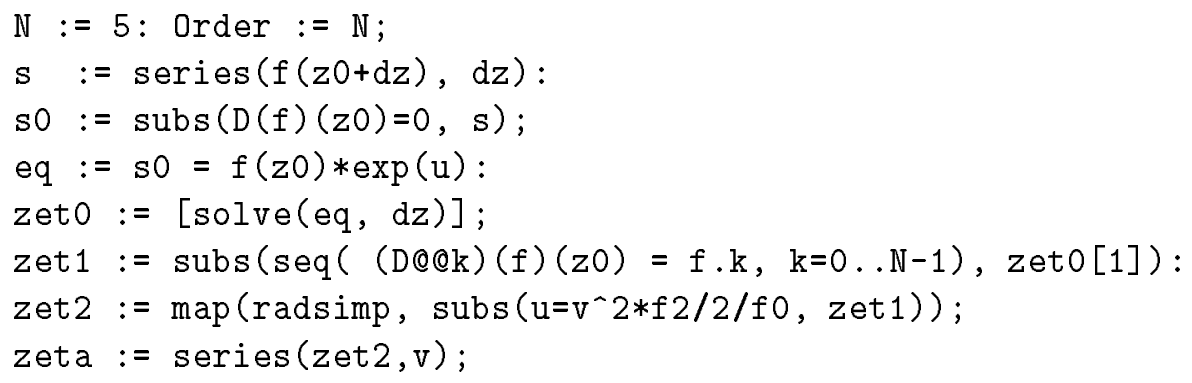

\footnotetext{
${ }^{2}$ The authors are indebted to Dominik Gruntz for this program.
} 
The output of this program contains the following lines:

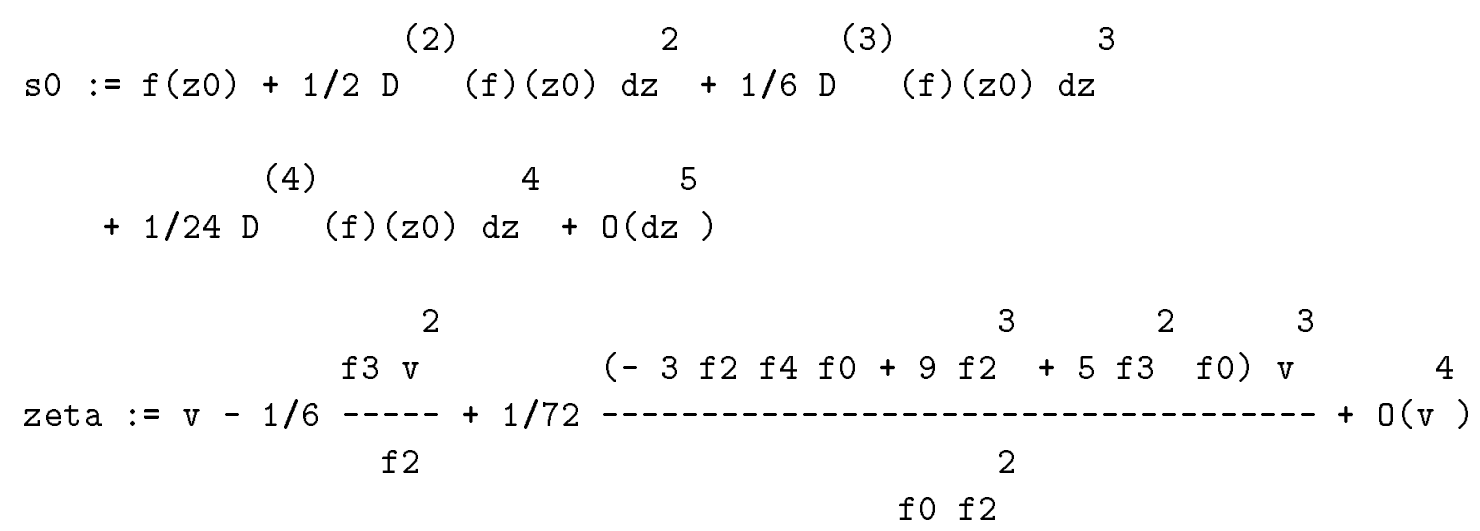

The MAPLE program works for any $N \geq 3$, producing $N-2$ terms of the above series. However, it is fairly slow, since no "intelligence", such as information on the form of the resulting series, is built in. To be able to find this series, nevertheless, is a good accomplishment of a general-purpose symbolic manipulator. It can be seen that $\zeta$ may be written as a formal power series in the variable $v$ defined in (13). If the original series (11) converges in a neighborhood of $z_{0}$, the resulting series converges in a neighborhood of $v=0$.

The directions $\theta_{0}$ of the $r$-lines at $z_{0}$ are now given by the values of $\zeta$ corresponding to $u=i t$ in the limit $t \rightarrow 0$. The above series and Equ. (13) immediately yield

$$
\theta_{0}=\arg v=\frac{1}{2}\left(\arg f_{0}-\arg f_{2} \pm \frac{\pi}{2}\right)
$$

Hence there are two $r$-lines through $z_{0}$ intersecting at right angles, in perfect agreement with Equ. (8).

The directions of the $\varphi$-lines through $z_{0}$, on the other hand, are given by (13) for real values of $u$. We obtain the two directions $\theta_{0} \pm \frac{\pi}{4}$, i.e., the tangents of the two $\varphi$-lines through $z_{0}$ are the bisectors of the tangents of the $r$-lines.

\section{The Contour Lines $r=1$ of $f=e_{n}$}

As indicated in $\S 3.1$, we need to solve (7) with initial values $x=y=0$. Let $\mathrm{S}_{\mathrm{n}}$ be the point of intersection of the 1-line of $e_{n}$ with the negative real axis. By symmetry, only the portion of each 1-line lying in the upper half of the complex plane needs to be computed. This is not quite easy in MATLAB, since the ode routine requires a terminal value $s_{f}$ of the independent variable $s$ and (unfortunately) does not allow termination of the integration process according to some condition (such as $y \geq 0$ ) being violated. One way to deal with this problem is to select $s_{f}$ as an upper bound of the arc length on the 1-line between the origin and $\mathrm{S}_{\mathrm{n}}$. Then, only the points satisfying the condition $y \geq 0$ need to be plotted. The point $S_{n}$ can be approximated by linear interpolation between the two points on the 1-line closest to $S_{n}$.

In the MATLAB program below this is done by using the find command with the parameter $y \geq 0$ in order to find the subset of all points satisfying the condition $y \geq 0$. Their indices are collected in the vector indices. The indices of the points used in linear interpolation are then $1=l$ ength (indices) and $11=1+1$. Finally, $w$ is the normalized row vector containing the two interpolation weights, and the actual interpolation is carried out by the product $w * z(1: 11,:)$. 
A good upper bound $s_{f}$ for the arc length is obtained from the observation that the region $\left|e_{n}(z)\right| \leq 1$ approaches a semidisk of radius $\rho(n)$ as $n \rightarrow \infty$. An asymptotic analysis shows that

$$
\rho(n)=\exp (-1) \cdot(n+\log \sqrt{2 \pi n}+O(1)) .
$$

It suffices to choose $O(1)=3$; then we obtain

$$
s_{f} \sim\left(1+\frac{\pi}{2}\right) \exp (-1) \cdot(n+\log \sqrt{2 \pi n}+3)
$$

as a close upper bound for the arc length up to the point $S_{n}$.

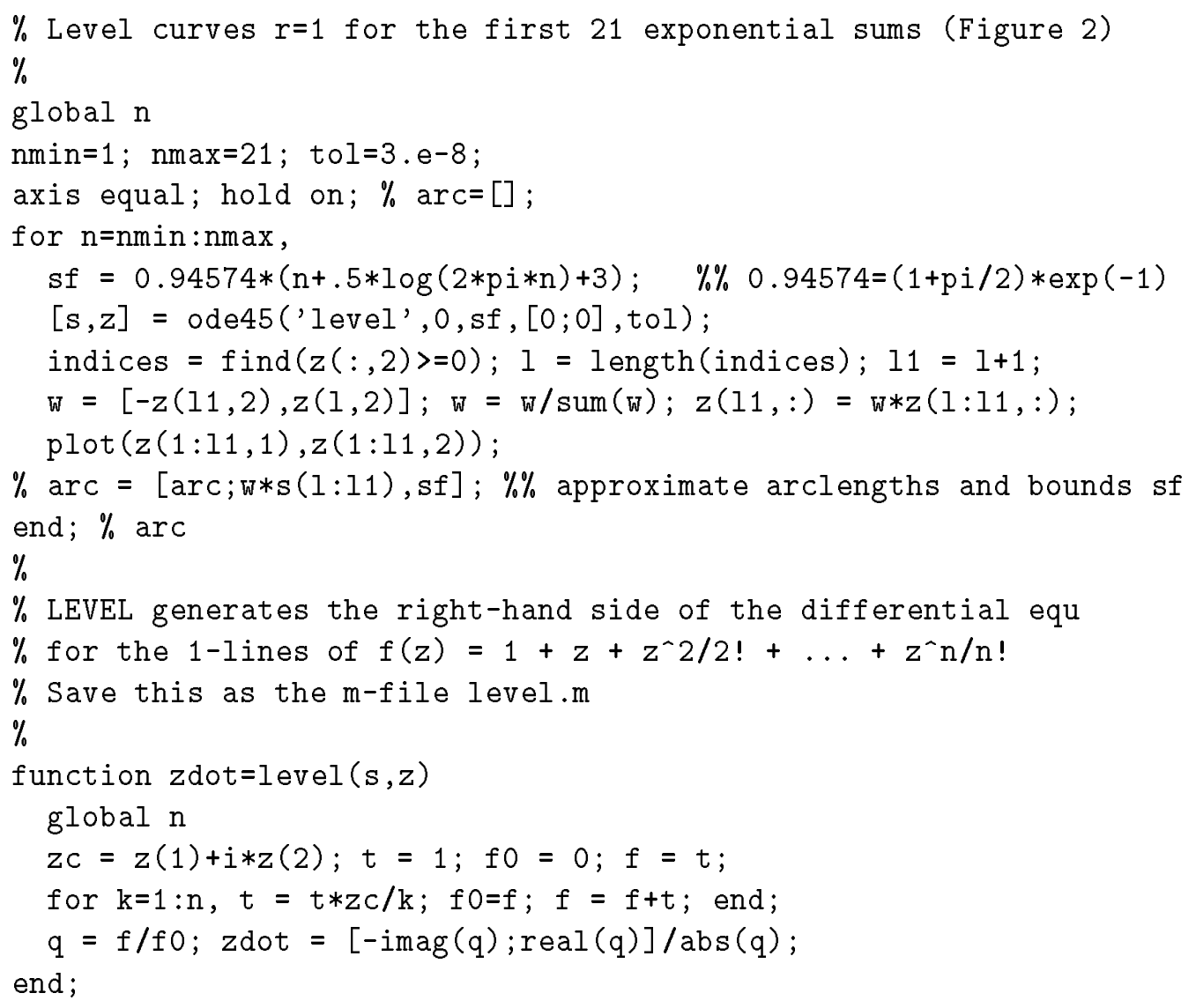

The MATLAB program begins with the definitions of the parameters nmin, nmax and the error tolerance tol. The 1-lines in the range nmin $\leq n \leq \mathrm{nmax}$ are generated in the accuracy given by tol and plotted. Rerunning the program with new values of nmin and nmax adds new curves to the figure. The statements turned off by the comment marks \% generate a table arc containing the actual arc lengths and the upper bounds $s_{f}$ computed from (14).

The actual integration is done in the call to the integrator ode45. The input parameters of this procedure are: the name 'level' (in string quotes) of the m-file defining the differential equations (7) to be integrated, the initial value 0 and the final value sf of the independent variable, the column vector $[0 ; 0]$ of the inital values, and (optionally) the error tolerance tol. The choice tol $=3.0 e-8$ yields a high-resolution plot, whereas the default tol $=1.0 e-6$ (when the parameter is omitted in the call) still yields a satisfactory plot. The values of the independent and dependent variables generated by the integrator are stored as the vectors $\mathbf{s}$ and $\mathbf{z}$, respectively, ready to be plotted. 
The results for $n=1: 1: 21$ are shown in Figure 2 below. The features near the imaginary axis at the transition to the circular part seem to show a periodicity in $n$ of a little over 5 . For example, the curves corresponding to $n=5,10,15,21$ all show a particularly large protrusion into the right half-plane.

An investigation of this phenomenon is interesting, but exceeds the scope of this article. We limit ourselves to reporting that as $n \rightarrow \infty$, the period tends to

$$
\frac{2 \pi}{\frac{\pi}{2}-\exp (-1)}=5.22329130 \text {. }
$$

This result was obtained by considering the function $e_{\nu}(z)$ for real values of $\nu$ (which leads to the incomplete gamma function) and requiring the 1-line of $e_{\nu}(z)$ to contain a saddle point with $\epsilon_{\nu}^{\prime}(z)=0$.

Figure 2. Level Curves $r=1$ for the First 21 Exponential Sums

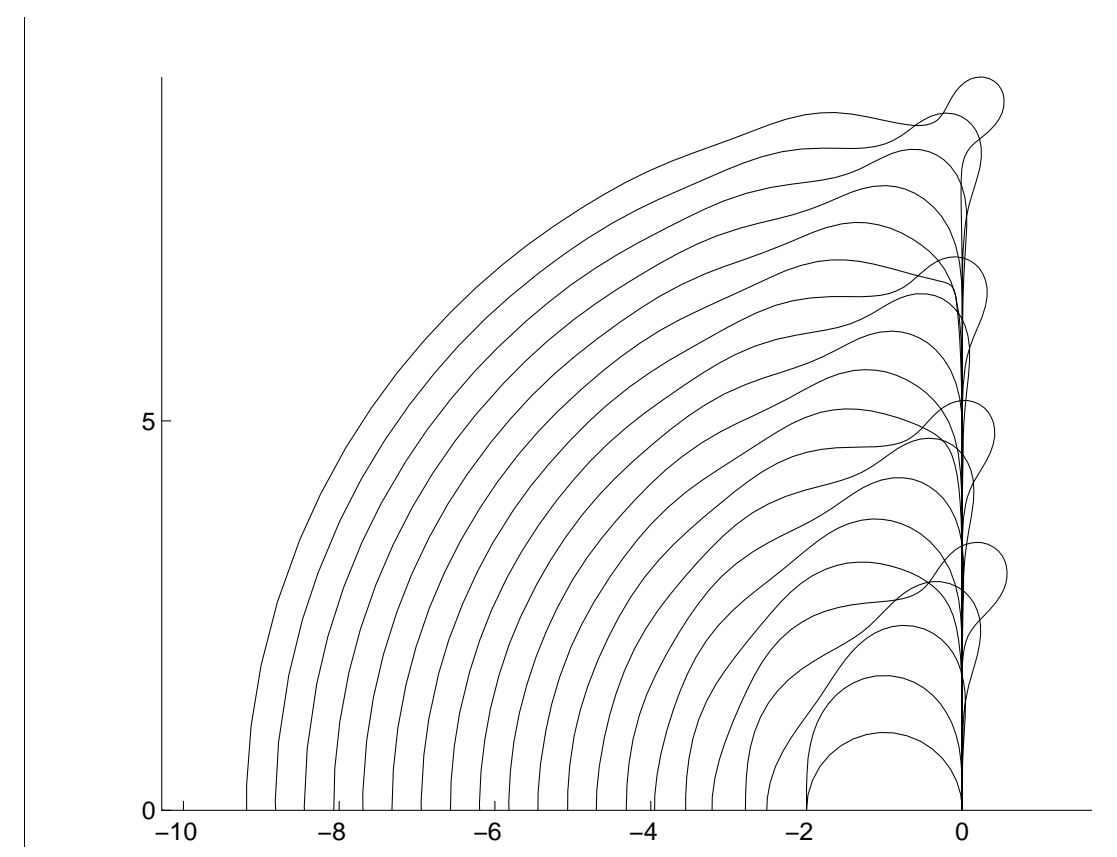

\section{The Contour Lines $\varphi=$ const of $f=e_{n}$}

Below is a MATLAB program that implements the method of $\S 3.2$ for any fixed $n>0$, where the differential equations (9) must be implemented in the function phase and stored in the $\mathrm{m}$-file phase.m. The program begins with the definitions of $n$, the error tolerance tol, and the desired arc length sf of the curve segments emanating from the zeros. Then, the vector $r$ of the zeros of $e_{n}$ is computed by means of the function roots, where the coefficients of $e_{n}$ are generated by means of the gamma function. On the next line the subset of the zeros in the upper half-plane is formed by means of the find command with the argument $\operatorname{imag}(r)>=0$. The statement used in the program stores all the indices defining the subset in the vector indices; then $r$ (indices) is the vector of the zeros of $e_{n}$ in the upper half-plane (which is printed for convenience).

The input parameters in the call to the integrator ode45 are: the name 'phase' (in string quotes) of the differential equations to be integrated, the initial value 0 and the final value 
sf of the independent variable, the column vector $z 0$ of the inital values, and (optionally) the error tolerance tol (default $10^{-6}$ when omitted). The values of the independent and dependent variables generated by the integrator are stored as the vectors $\mathbf{s}$ and $\mathbf{z}$, respectively, ready to be plotted.

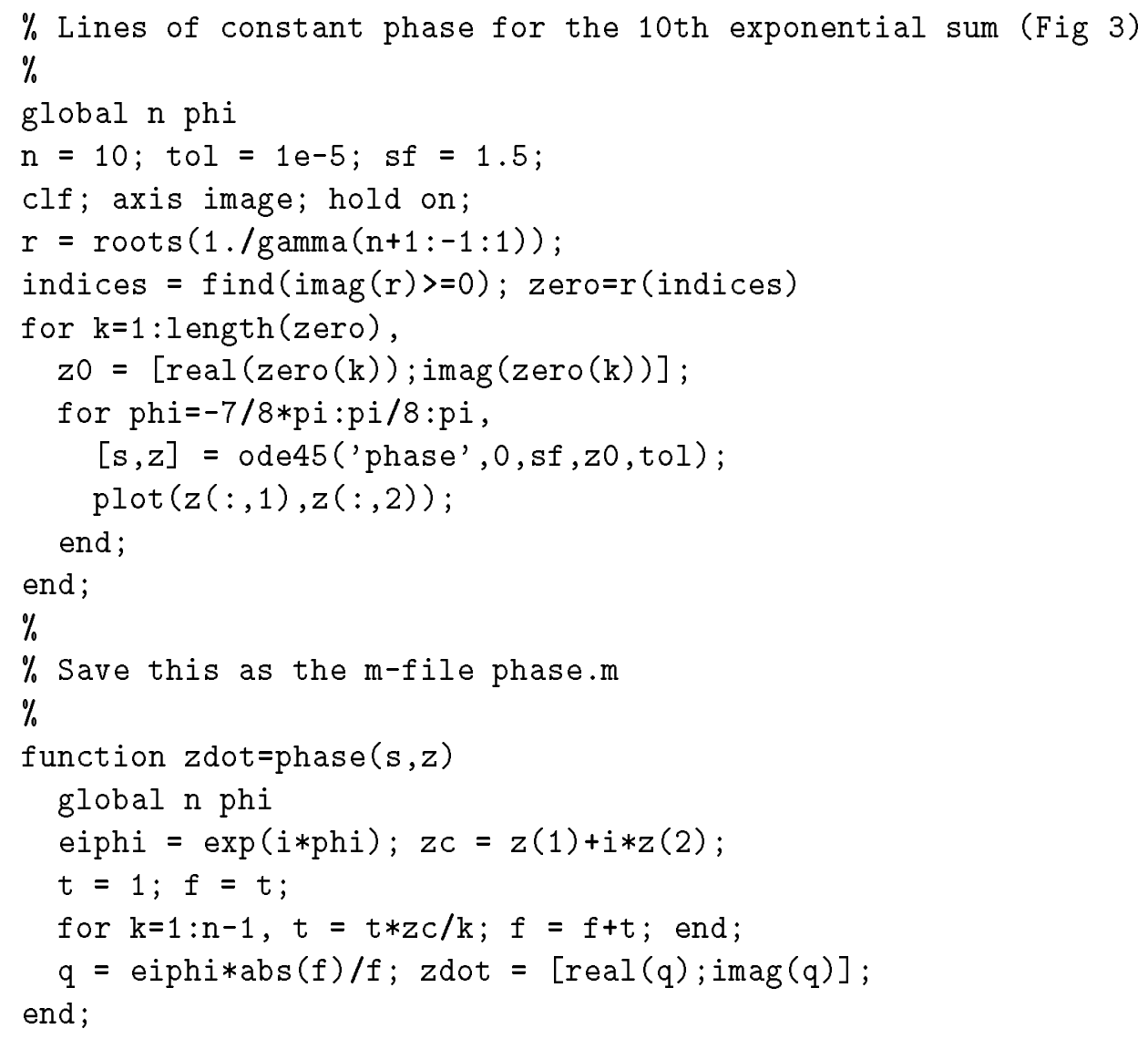

The result for $n=10$ is shown in Figure $3^{3}$.

Figure 3. Lines of Constant Phase for the 10th Exponential Sum

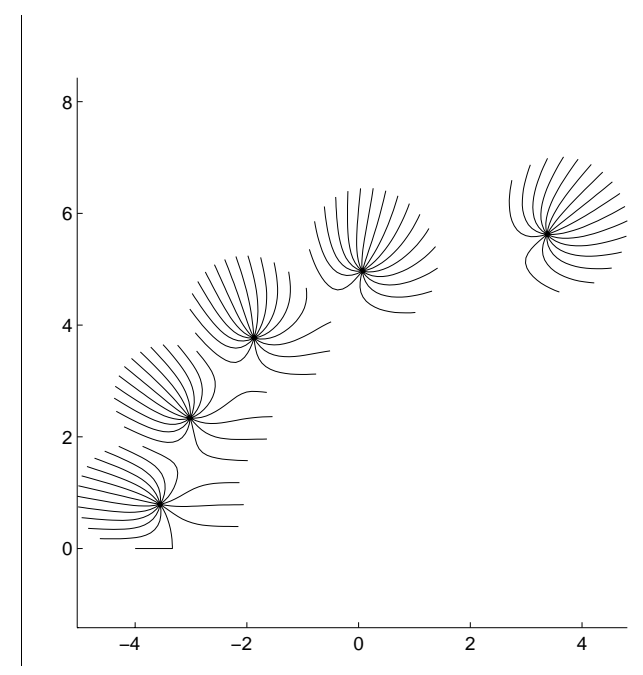

\footnotetext{
${ }^{3}$ We wrote and ran the script on August 1, 1996, while fireworks went off in celebration of the Swiss national holiday.
} 
None of the complex singular points is in evidence in Figure 3 since the $\varphi$-values chosen do not correspond to level lines passing through a complex singular point. The real singular point at $z_{0}=-3.333551485$, however, is clearly visible by an abrupt right-angled turn of the line $\varphi=0$ (near the bottom of the figure). It is curious to note how the ode 45 integrator was able to integrate right through the singularity, or so it seems.

\section{References}

[1] A.J. Carpenter, R.S. Varga, J. Waldvogel, Asymptotics for the zeros of the partial sums of $e^{z} . I$, Rocky Mountain J. Math., 21, 1991, pp. 99-120.

[2] K.E. Iverson, The zeros of the partial sums of $e^{z}$, Math. Tables and Other Aids to Computation, 7, 1953, pp. 165-168.

[3] G. Pólya, G. Szegö, Problems and Theorems in Analysis, Vol. II, Part Five, Exercise 74, Springer-Verlag, Berlin, 1976.

[4] H.R. Schwarz, Numerical Analysis. A Comprehensive Introduction, John Wiley \& Sons, Chichester, 1989. 